\title{
Nanoimaging for Protein Misfolding Diseases. Critical Role of Misfolded Dimers in the Amyloid Self-Assembly
}

\author{
Alexey V. Krasnoslobodtsev, Bo-Hyun Kim, and Yuri L. Lyubchenko* \\ Department of Pharmaceutical Sciences, University of Nebraska Medical Center, Omaha, NE \\ 68198-6025
}

Misfolding and aggregation of proteins are widespread phenomena leading to the development of numerous neurodegenerative disorders such as Parkinson's, Alzheimer's, and Huntington's diseases. Numerous lines of evidence suggest that the propensity to aggregate is considered a general characteristic of the majority of proteins. X-ray crystallography, NMR, electron microscopy, and AFM have provided important information on the structure of aggregates.

Despite the crucial importance of protein misfolding and spontaneous aggregation in disease, very little is known about the molecular mechanisms underlying these processes. Although it is well known that the same protein can form fibrillar, pore-like, spherical, or amorphous aggregates with diverse biological consequences, the conditions leading to misfolding and the formation of various complexes are unclear. Factors that facilitate protein misfolding and aggregation in vitro are poorly understood, not to mention the complexities involved in the formation of protein nanoassemblies with different morphologies, e.g., spherical oligomers and nanopores. Due to the difficulties in monitoring aggregates in their native state, the mechanisms underlying aggregation in biological systems in vivo are even less understood. A process that may appear simple by existing techniques may actually have many steps and a variety of important intermediate states - each with its own unique dynamics. Intermediate states are stabilized by weak interactions that are typically transient and difficult to measure. However, fundamental questions, such as why the misfolded conformation of the protein is formed, and why this state is important for self-assembly, remain unanswered. Misfolded states exist transiently, so answering these questions requires the use of novel approaches and methods. The lack of efficient methods to characterize the complex aggregation process is the major reason for our limited knowledge.

We developed a novel experimental approach that can directly probe aggregation-prone states of proteins and their interactions [1-5]. In this approach, the proteins are anchored to the surfaces of the AFM substrate (mica) and the probe (Fig. 1A), and the interaction between such anchored molecules is measured in the approach-retraction cycles (Fig. 1A-C). The forces stabilizing the complex are measured at the rupture event (Fig. 1C). The rupture force is low if the complex is weak (normal state of the protein; Fig. 1D; the force curve is shown as inset "d" in this scheme), but the forces increase if the protein adopts a misfolded conformation as illustrated in Fig. 1E and inset "e" in this scheme. AFM experiments with different proteins confirmed this relationship, demonstrating a direct correlation between the propensity of a protein to aggregate and the strength of the interprotein interaction forces $[1,2,6]$.

Using this approach, we were able to identify misfolded states of protein and characterize their property during interaction [7-9]. The major finding is that a misfolding dimer is characterized by a long lifetime that is enormously higher compared to the fast conformational dynamics of monomers that occurs in the microsecond-nanosecond time scale. A high stability of misfolded 
dimers is a fundamental finding, suggesting that the formation of dimers leads to huge stabilization of the protein misfolded state. Such stabilization dramatically facilitates the aggregation kinetics and thus triggers the entire aggregation process. This conclusion is in line with recent findings on the critical role of dimers in the self-assembly of proteins. Importantly, recent in vivo studies revealed a high toxicity of dimeric forms of amyloid beta. Further applications of these studies will be discussed.

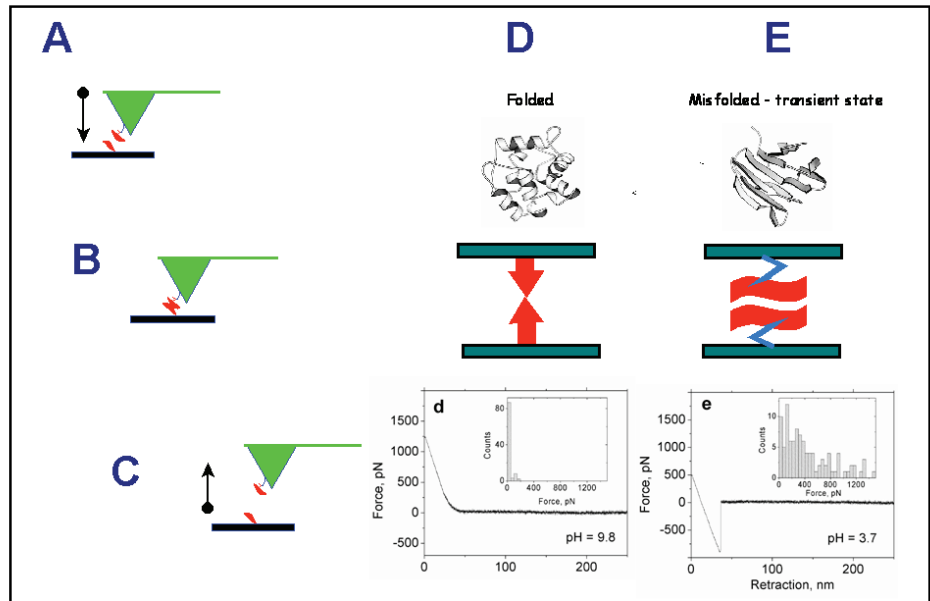

Figure 1. Scheme explaining the nanoprobing approach for detecting and analyzing misfolded states of the protein. Proteins anchored to the substrate surface and the AFM tip $(A)$ are brought into contact $(B)$ and then pulled apart $(C)$. The rupture forces of the complexes depend on the protein conformation. They are low for normally folded proteins (the force curve "d"). Large rupture force (e) corresponds with a misfolded state of the protein $(E)$.
*Correspondence to: Lyubchenko Y. L. Department of Pharmaceutical Sciences, University of Nebraska Medical Center, Omaha, NE 68198. Email: ylyubchenko@unmc.edu

This research was supported by grants: DOE (DE-FG02-08ER64579), NATO (CBN.NR.NRSFP 983204), NRI-07 and NIH (GM096039) (to YLL).

\section{References}

[1]. Kransnoslobodtsev, A. V., Shlyakhtenko, L. S., Ukraintsev, E., et al., Nanomedicine 1(2005), 300-05.

[2]. McAllister, C., Karymov, M. A., Kawano, Y., et al., J Mol Biol 354(2005), 1028-42.

[3]. Lyubchenko, Y. L., Sherman, S., Shlyakhtenko, L. S., et al., J Cell Biochem 99(2006), $52-70$

[4]. Yu, J., Malkova, S., and Lyubchenko, Y. L., J Mol Biol 384(2008), 992-1001.

[5]. Yu, J., and Lyubchenko, Y. L., J Neuroimmune Pharmacol 4(2009), 10-6.

[6]. Lyubchenko, Y. L., Sherman, S., Shlyakhtenko, L. S., et al., J Cell Biochem 99(2006), 53-70.

[7]. Krasnoslobodtsev, A. V., Portillo, A. M., Deckert-Gaudig, T., et al., Prion 4(2010).

[8]. Lyubchenko, Y. L., Kim, B. H., Krasnoslobodtsev, A. V., et al., Wiley Interdiscip Rev Nanomed Nanobiotechnol 2(2010), 526-43.

[9]. Yu, J., Warnke, J., and Lyubchenko, Y. L., Nanomedicine in press(2010). 